\title{
ROBUST TASK SPACE TRAJECTORY TRACKING CONTROL OF ROBOTIC MANIPULATORS
}

\author{
M. GALICKI \\ Faculty of the Mechanical Engineering \\ University of Zielona Góra \\ ul. Prof. Z. Szafrana 4, 65-516, Zielona Góra, POLAND \\ E-mail: M.Galicki@ibem.uz.zgora.pl
}

\begin{abstract}
This work deals with the problem of the accurate task space trajectory tracking subject to finite-time convergence. Kinematic and dynamic equations of a redundant manipulator are assumed to be uncertain. Moreover, globally unbounded disturbances are allowed to act on the manipulator when tracking the trajectory by the end-effector. Furthermore, the movement is to be accomplished in such a way as to reduce both the manipulator torques and their oscillations thus eliminating the potential robot vibrations. Based on suitably defined task space non-singular terminal sliding vector variable and the Lyapunov stability theory, we propose a class of chattering-free robust controllers, based on the estimation of transpose Jacobian, which seem to be effective in counteracting both uncertain kinematics and dynamics, unbounded disturbances and (possible) kinematic and/or algorithmic singularities met on the robot trajectory. The numerical simulations carried out for a redundant manipulator of a SCARA type consisting of the three revolute kinematic pairs and operating in a two-dimensional task space, illustrate performance of the proposed controllers as well as comparisons with other well known control schemes.
\end{abstract}

Key words: redundant manipulator, task space trajectory cracking, finite-time control, Lyapunov stability, vibration reduction.

\section{Introduction}

Robotic (redundant) manipulators have been widely employed for decades in industry. They have found a broad area of applications which include inserting a shaft into a bearing hole or an assembly of electronic components onto the small surface of printed circuit boards. These tasks require, by their nature, extremely high precision and stability of the performance. Therefore, it is particularly important to design control strategies that fulfil the aforementioned requirements despite the uncertainties of both robot kinematic and dynamic equations, unknown payloads to be transferred by the end-effector as well as external disturbances. In most situations met in practice, those tasks are specified in terms of a trajectory expressed in Cartesian (task) coordinates to be tracked by the end-effector. On the other hand, redundant degrees of freedom make it possible to achieve some useful objectives such as collision avoidance in the work space with obstacles, joint limit avoidance and/or reaching some rest (preferred) posture. Thus, a controller to be designed for kinematically redundant manipulators should both accurately track desired end-effector trajectory (primary task) and accomplish additive objectives (secondary task) subject to uncertain both kinematic and dynamic equations and external disturbances. Due to the challenging nature of the aforementioned control design problems, many researchers have proposed different types of controllers. In such a context, one can distinguish a few approaches of controlling the kinematically redundant manipulators. The control techniques offered in Khatib, [1]; Hsu et al. [2]; Canudas, et al. [3]; Siciliano, et al. [4]; Galicki, [5]; Kelly and Moreno, [6]; Nakanishi, et al. [7]; Moreno-Valenzuela and Gonzales-Hernandez, [8] require the full knowledge of the robot dynamics neglecting the external disturbances. Works Tatlicioglu et al. [9]; Sadeghian et al. [10]; Sadeghian et al. [11]; Feng and Palaniswami, [12]; Zergeroglu et al. [13]; Braganza et al. [14]; Braganza et al. [15]; Galicki, [16]; Cheah et al. [17]; Li and Cheah, [18]; Li and Cheah, [19]; 
Galicki, [20]; Zuo, [21] present adaptive control algorithms to compensate for parametric uncertainties in dynamic model including only the linearly parameterizable friction terms (viscous friction) and also neglecting the external (non-linearly parameterizable) disturbances. Moreover, control laws from Tatlicioglu et al. [9]; Sadeghian et al. [10]; Sadeghian et al. [11]; Feng and Palaniswami, [12]; Zergeroglu et al. [13]; Braganza et al. [14]; Braganza et al. [15]; Galicki, [16]; Cheah et al. [17]; Li and Cheah, [18]; Li and Cheah, [19] use pseudo-inverse of either the exact or approximate Jacobian matrices. Our recent study Galicki, [20] estimates the pseudo-inverse by some non-singular matrix which is adaptively computed. Model based robust control schemes were investigated in works Zergeroglu et al. [22]; Ozbay et al. [23]; Singh and Sukavanam, [24]; Nandhakumar et al. [25]. The controllers proposed in Zergeroglu et al. [22]; Ozbay et al. [23] ensure uniformly ultimately bounded end-effector and sub-task tracking despite the parametric uncertainties associated with the dynamic equations, an upper bound on the parameter accuracy and globally bounded external disturbances. A neural network based adaptive asymptotically stable control scheme, in the presence of model uncertainties and globally bounded external disturbances, has been designed in Singh and Sukavanam, [24]. A fuzzy logic, adapted to transpose Jacobian control law by tracking a Cartesian trajectory, has been proposed in work Green and Sasiadek, [26]. The succeeding approach presented in only few works Seraji and Colbaugh, [27]; Peng and Adachi, [28]; Ott et al. [29]; Oh and Chung, [30]; Colbaugh and Glass, [31] is based on utilizing augmented task space techniques such as the extended Jacobian Balleieul, [32] or the configuration control proposed by Seraji and Colbaugh, [27]. In this approach, the dimension of the operational space is extended by incorporating as many additional constraints as the degrees of redundancy, and hence, the resulting system becomes non-redundant. The control algorithms from Seraji and Colbaugh, [27]; Peng and Adachi, [28]; Ott et al. [29] require exact dynamic model thus guaranteeing the asymptotic tracking. Using extended Jacobian, nominal values of the parameters of dynamic equations and the momentum feed-back disturbance observer, a trajectory tracking control law has been proposed in Oh and Chung, [30] without its stability analysis. Work Colbaugh and Glass, [31] presents an adaptive scheme for the motion control of kinematically redundant manipulator subject to parametric uncertainties and globally bounded disturbances. In the absence of disturbances and under some sufficient conditions on gain parameters, control law from Colbaugh and Glass, [31] is shown to ensure only globally ultimately bounded Cartesian tracking error. From the literature survey, it follows that all the aforementioned control schemes require either the knowledge of the nominal robot dynamic equations whose construction may not be a trivial task or involve all the adaptive terms multiplied by the regression matrix (neglecting the external non-linearly parameterizable disturbances) that seems to be both complex to implement and very time consuming. Control algorithms based on the pseudoinverse provide only sub-optimal (and not optimal) solutions and are not, in general, repeatable Shamir and Yomdin, [33]; Roberts and Maciejewski, [34]. Consequently, an important class of cyclic technological operations (cyclic kinematic tasks) cannot be accomplished by this approach. Moreover, almost all the trajectory tracking algorithms require explicitly inverse or pseudo-inverse of a Jacobian matrix or its estimate which can potentially contain kinematic and/or algorithmic singularities. As a consequence, these algorithms can cause the control inputs to become unbounded what leads to vibrations of the robot. Furthermore, all the control schemes assume globally bounded disturbances when tracking the trajectory whereas e.g. a viscous friction term is globally unbounded with respect to joint velocity. The assumption of global boundedness of external disturbances may lead to deterioration of the accuracy of trajectory tracking. Finally, aforementioned controllers provide only at most asymptotic stability what may be insufficient for accomplishment of tasks requiring the extremely high precision (e.g. assembly of electronic components on the small surface of printed circuit boards).

In this paper, we propose a robust controller for kinematically redundant manipulators using an augmented task space formulation. Motivated in part by the computed torque joint space based approach Spong and Vidyasagar, [35]; Siciliano, et al. [4], we introduce a new class of robust controllers based on estimate of the transpose Jacobian. The advantages of the estimated Jacobian transpose approach are that it is computationally efficient and it does not suffer from singularity problem. Moreover, the proposed control laws ensure both optimal solution and a cyclic joint trajectory provided the desired end-effector trajectory is cyclic. As opposed to other existing robust control algorithms, our approach both reduces the values of torques by minimizing a suitably chosen objective function and eliminates their oscillations by introducing a boundary layer technique. 
The boundary layer technique, introduced herein to eliminate undesirable chattering effect, guarantees arbitrarily small bounds on the tracking errors without increase of gain coefficients. The remainder of the paper is organised as follows. Section 2 formulates the finite-time trajectory tracking task. Section 3 sets up a class of task space robust controllers solving the trajectory tracking problem in a finite-time subject to uncertain robot dynamic equations and unbounded disturbances. Section 4 presents computer examples of the endeffector trajectory tracking by a redundant manipulator of a SCARA type, consisting of three revolute kinematic pairs and operating in a two-dimensional task space. Finally, some concluding remarks are drawn in Section 5.

\section{Problem formulation}

The robust control scheme designed in the next section is applicable to holonomic fully actuated mechanical systems comprising redundant manipulators considered here which are described, in general, by the following dynamic equations, expressed in generalized (joint) coordinates $q=\left(q_{1}, \ldots, q_{n}\right)^{T}$ Spong and Vidyasagar, [35],

$$
M(q) \ddot{q}+C(q, \dot{q}) \dot{q}+G(q)+D(t, q, \dot{q})=v
$$

where $n$ is the number of kinematic pairs of the V-th class; $\dot{q}$ and $\ddot{q}$ represent velocity and acceleration, respectively expressed in generalized coordinates; $M(q)$ denotes the $n \times n$ positive definite, symmetric inertia matrix; $C(q, \dot{q}) \dot{q}$ is the $n$-dimensional vector representing centrifugal and Coriolis forces; $v=\left(v_{1}, \ldots, v_{n}\right)^{T}$ stands for the $n$-dimensional vector of controls (torques/forces); $G(q)$ is the $n$-dimensional vector of generalized gravity forces; $D(t, q, \dot{q})$ means the $n$-dimensional external disturbance signal which is (by assumption) at least a locally bounded Lebesgue measurable mapping. Without loss of generality, $D$ is assumed to be upper estimated as follows

$$
\|D\| \leq \alpha_{0}(t, q, \dot{q})
$$

where \|\| denotes Euclidean norm; $\alpha_{0}(\cdot)$ stands for the time dependent known non-negative locally bounded Lebesgue measurable function. In what follows, $D$ is assumed in further analysis to represent both the friction in the sliding (viscous) and in the presliding (also called stiction) regime Haessing and Friedland, [36]; Wit et al. [37]. In such a case, $D$ equals

$$
D=d_{0}(q-q(0))+d_{1} \dot{q}+d_{2} \operatorname{sign}(\dot{q})+d_{3} \exp \left(-d_{4}\|\dot{q}\|^{2}\right) \operatorname{sign}(\dot{q})
$$

where $q(0)$ means initial posture of the manipulator; $d_{0}$ denotes a stiffness coefficient for the position-friction torque relationship at joint velocity reversal; $d_{1}$ is a viscous damping coefficient; $d_{2}$ stands for the Coulomb coefficient and $d_{3}, d_{4}$ are constants representing the Stribeck effect.

Consequently

$$
\alpha_{0}=\overline{d_{0}}\|q-q(0)\|+\overline{d_{1}}\|\dot{q}\|+\overline{d_{2}}+\overline{d_{3}} \exp \left(-\underline{d_{4}}\|\dot{q}\|^{2}\right)
$$


where $\overline{d_{0}}, \ldots, \overline{d_{3}}$ stand for upper estimations of unknown coefficients $d_{0}, \ldots, d_{3}$ and $\underline{d}_{4}$ denotes lower estimate of the Stribeck parameter $d_{4}$, respectively.

The direct kinematic mapping between joint coordinates $q$ of the manipulator and the end-effector coordinates $p_{e}=\left(p_{e, 1}, \ldots, p_{e, m}\right)^{T} \in \mathbb{R}^{m}$ can be written as

$$
p_{e}=f_{e}(q, X)
$$

where $m$ is the dimension of the Cartesian space in which the end-effector operates; $f_{e}: \mathbb{R}^{n} \times \mathbb{R}^{k} \rightarrow \mathbb{R}^{m}$ denotes the $m$-dimensional non-linear (with respect to $q$ ) mapping constructed from the kinematic equations of the manipulator; $f_{e}=\left(f_{e, 1}, \ldots, f_{e, m}\right)^{T} ; X=\left(X_{1}, \ldots, X_{k}\right)^{T}$ stands for an ordered set of kinematic parameters such as link lengths and/or joint offsets; $k$ denotes the number of kinematic parameters. Moreover, there exist upper $X_{u}$ and lower $X_{l}$ bounds, respectively such that

$$
X_{l} \leq X \leq X_{u}
$$

On account of the redundant manipulator considered herein, the relation $n>m$ holds. Its consequence is a possibility to augment the end-effector conventional trajectory tracking (primary task) with additional user-specified useful task coordinates $p_{a}=\left(p_{a, l}, \ldots, p_{a, n-m}\right)^{T} \in \mathbb{R}^{n-m}$ (secondary task) of the following general form

$$
p_{a}=f_{a}(q, X)
$$

where $f_{a}: \mathbb{R}^{n} \times \mathbb{R}^{k} \rightarrow \mathbb{R}^{n-m}$ is a given at least twice differentiable mapping with respect to $q$; $f_{a}=\left(f_{a, 1}, \ldots, f_{a, n-m}\right)^{T}$. From the practical point of view, it is particularly important to generate joint trajectory $q=q(t)$ in such a way as to minimize an objective function $\mathcal{A}(q, X)$ subject to constraints Eq.(2.5). The objective function $\mathcal{A}$ may be any at least twice differentiable mapping representing a measure of some kinematic characteristic of the manipulator performance so that the redundant degrees of freedom are exploited to fulfil additional goals: a singularity avoidance, posture control, obstacle avoidance, etc. Yoshikawa, [38]; Maciejewski and Klein, [39]. The general form for $f_{a}$, proposed, e.g., in works Balleieul, [32]; Perdereau et al. [40] may be expressed as

$$
f_{a}=\mathcal{N}(q, X) \frac{\partial \mathcal{A}(q, X)}{\partial q}
$$

where $\mathcal{N}$ stands for the $(n-m) \times n$ orthogonal complementary matrix to $J(q, X)=\frac{\partial f_{e}(q, X)}{\partial q}$, i.e., ${ }^{\prime} \mathcal{N}^{T}=0$. Without loss of generality, we shall employ a simple and practically useful optimization criterion for redundancy resolution with a cost function

$$
\mathcal{A}(q)=\frac{c_{\mathcal{A}}}{2}\left\langle q-q_{\text {rest }}, K_{\mathcal{A}}\left(q-q_{\text {rest }}\right)\right\rangle
$$


where $\langle$,$\rangle denotes the scalar product of vectors; c_{\mathcal{A}}$ is a positive constant; $q_{\text {rest }}$ stands for some rest (preferred) posture; $K_{\mathcal{A}}$ is a positive definite diagonal weighting matrix. This criterion has been shown to be useful when creating human-like movement in anthropomorphic robots (Cruse et al. [41]. Moreover, for $q_{\text {rest }}=q(0)$, minimization of criterion Eq.(2.9) results in avoiding sudden changes of manipulator configurations and consequently allows to reduce both the values of torques in $L_{2}$ norm and potential robot vibrations by appropriate weighting of joints closer to the robot base.

Concatenating Eq.(2.5) with Eq.(2.8) yields the general kinematic and differential mappings between $q$ and extended task coordinates $p=\left(p_{e}^{T} p_{a}^{T}\right)^{T}$

$$
p=f(q, X), \quad \dot{p}=J(q, X) \dot{q}
$$

where $f=\left(f_{e}^{T}\left(\mathcal{N} \frac{\partial \mathcal{A}}{\partial q}\right)^{T}\right)^{T}$ and $J=\frac{\partial f}{\partial q}$ is the $n \times n$ extended Jacobian matrix.

A task accomplished by the redundant manipulator consists in tracking both a desired end-effector trajectory $p_{d}^{e}(t) \in \mathbb{R}^{m}, t \in[0, \infty)$ which is assumed to be at least twice continuously differentiable, i.e., $p_{d}^{e}(\cdot) \in C^{2}[0, \infty)$ and desired user specified trajectory $p_{d}^{a}(t) \in \mathbb{R}^{n-m}$ which equals $p_{d}^{a}(t)=0$.

By introducing the task tracking error $e=\left(e_{1}, \ldots, e_{n}\right)^{T}=\left(\left(e^{e}\right)^{T}\left(e^{a}\right)^{T}\right)^{T}=f(q, X)-p_{d}(t)$, where $p_{d}=\left(\left(p_{d}^{e}\right)^{T}(0)^{T}\right)^{T} ; e^{e}=f_{e}-p_{d}^{e} ; e^{a}=\mathcal{N} \frac{\partial \mathcal{A}}{\partial q}$, we may formally express the task space finite-time control problem by means of the following equations

$$
\begin{array}{ll}
\lim _{t \rightarrow T} \quad e(t)=0, \\
\lim _{t \rightarrow T} \quad \dot{e}(t)=0
\end{array}
$$

where $0 \leq T$ denotes a finite-time of convergence of $f(q)$ to $p_{d} ; e(t)=\dot{e}(t)=0$ for $t \geq T$. Observe that upper equation of Eq.(2.11) presents for $t \geq T$ a necessary condition for minimum of $\mathcal{A}$ subject to equality constraint $f_{e}-p_{d}^{e}=0$. Let us also note that fulfilment of Eq.(2.11) makes a repetitive motion conservative, i.e., closed end-effector trajectory $p_{d}^{e}$ in task space forces the corresponding closed trajectory in the joint space Shamir and Yomdin, [33]; Roberts and Maciejewski, [34]. This property seems to be very useful in accomplishing the cyclic technological operations. Let us rewrite Eq.(2.1) in a more compact form as follows

$$
M(q) \ddot{q}+F(t, q, \dot{q})=v
$$

where $F=C \dot{q}+G+D$. In the sequel, useful properties of Eq.(2.1), Eq.(2.12) are summarised which will be utilised while designing the controller. The following inequalities are satisfied Spong and Vidyasagar, [35] 


$$
\begin{aligned}
& 0<\left\|M^{-1}\right\|_{F} \leq \Lambda_{\max }, \\
& \|C \dot{q}\| \leq c_{1}\|\dot{q}\|^{2} \\
& \|G\| \leq c_{2}
\end{aligned}
$$

where \|\|$_{F}$ means Frobenius (Euclidean) matrix norm; $\Lambda_{\max }, c_{1}, c_{2}$ are known positive scalar coefficients. Based on the properties of Eq.(2.13), one obtains the following upper estimation of $F$

$$
\|F\| \leq \mathcal{E}(t, q, \dot{q})
$$

where $\varepsilon=c_{1}\|\dot{q}\|^{2}+c_{2}+\alpha_{0}$. Moreover, on account of Eq.(2.6), Eq.(2.8), Eq.(2.9) and definition of $J$ in Eq.(2.10), the following inequalities hold true for revolute kinematic pairs

$$
\begin{aligned}
& \|J\|_{F} \leq c_{3}+c_{4}\left\|q-q_{\text {rest }}\right\|, \\
& \left\|\frac{\partial J}{\partial q}\right\|_{F} \leq c_{5}+c_{6}\left\|q-q_{\text {rest }}\right\|
\end{aligned}
$$

where $\|J\|_{F}=\max _{X_{l} \leq X \leq X_{u}}\left\{\|J(q, X)\|_{F}\right\} ; c_{3}, \ldots, c_{6}$ are known scalar coefficients. Based on relations (2.13)(2.15), the next section will present an approach to the solution of the control problem (2.1), (2.11) and (2.12) making use of the Lyapunov stability theory.

\section{Task space control of the robotic redundant manipulator}

Motivated in part by the static computed torque methodology Spong and Vidyasagar, [35]; Siciliano, et al. [4], we propose now a new control law with transpose estimated Jacobian of the form

$$
v=\hat{J}^{T} u
$$

where $\hat{J}=J(q, \hat{X})$ estimates the uncertain Jacobian matrix $J=J(q, X) ; \hat{X}$ denotes estimation of the unknown kinematic parameters $X ; X_{l} \leq \hat{X} \leq X_{u} ; u \in \mathbb{R}^{n}$ is a new control to be determined. In further analysis, $\hat{J}$ is assumed to be of the full rank in the operation region of the end-effector.

Consequently, one also obtains that

$$
0<a \mathbb{I}_{n} \leq \hat{J} M^{-1} \hat{J}^{T}
$$

where $a$ denotes estimation of the minimal eigenvalue of matrix $\hat{J} M^{-1} \hat{J}^{T} ; \mathbb{I}_{n}$ stands for the $n \times n$ identity matrix.

The objective is to find input signal $u(t)$ and consequently control vector $v(t)$, such that end-effector location vector $p$ follows $p_{d}$. For this purpose, let us twice differentiate $e$ with respect to time thus obtaining 


$$
\ddot{e}=J M^{-1} \hat{J}^{T} u+Q-\ddot{p}_{d}
$$

where $Q=-J M^{-1} F+\dot{J} \dot{q}$. Furthermore, based on Eq.(2.14) and definition of $Q$ in Eq.(3.3), an upper estimation on $\|Q\|$ takes the form

$$
\|Q\| \leq \mathcal{W}(t, q, \dot{q})
$$

where $\mathcal{W}=\|J\|_{F} \cdot \Lambda_{\max } \cdot \mathcal{E}+\left\|\frac{\partial J}{\partial q}\right\|_{F} \cdot\|\dot{q}\|^{2}$. Let $s=\left(s_{1}, \ldots, s_{n}\right)^{T} \in \mathbb{R}^{n}$ be a task space sliding vector variable. In order to overcome the limitations and shortcomings of the first order classic sliding variables expressed in joint coordinates Feng et al. [42]; Yu et al. [43]; Zhao et al. [44], we propose the following non-singular terminal sliding manifold expressed in the task coordinates

$$
s=\dot{e}+\int_{0}^{t}\left(\lambda_{0} e^{\alpha_{1}}+\lambda_{l}(\dot{e})^{\alpha_{2}}\right) d \tau
$$

where $\alpha_{1}=\frac{a}{b} ; a, b$ are positive odd numbers, $a<b<2 a ; \quad \alpha_{2}=\frac{2 \alpha_{1}}{1+\alpha_{1}} ; \lambda_{0}=\operatorname{diag}\left(\lambda_{0,1}, \ldots, \lambda_{0, n}\right)$; $\lambda_{1}=\operatorname{diag}\left(\lambda_{1,1}, \ldots, \lambda_{1, n}\right) ; \lambda_{i, j} ;$ stand for positive coefficients (controller gains); $i=0: 1 ; j=1: n$. In what follows, we give a useful result Galicki, [45].

Lemma 1. If $s=0$ then task errors $(e, \dot{e})$ converge in a finite-time to the origin $(e, \dot{e})=(0,0)$. as follows

In order to fulfil equality constraints (2.11), a (simple) robust task space control law is proposed

$$
u(t, q, \dot{q}, e, \dot{e})= \begin{cases}-\frac{c}{a} \frac{s}{\|s\|}\left(\mathcal{V}(t, e, \dot{e})+\mathcal{W}(t, q, \dot{q})+c^{\prime}\right) & \text { for } s \neq 0 \\ 0 & \text { otherwise }\end{cases}
$$

where $\mathcal{V}=\left\|\lambda_{0} e^{\alpha_{1}}+\lambda_{1}(\dot{e})^{\alpha_{2}}-\ddot{p}_{d}\right\| ; c, c^{\prime}$ are positive constant gains specified follow $c>\frac{1}{1-5} ; \delta$ is a gain coefficient; $\delta \in(0,1) ; c^{\prime}>0$ and $\lambda_{0}, \lambda_{1}>0$.

Consequently, vector $v$ can be found from the following equation with $u$ given by Eq.(3.6)

$$
v=J^{T}(q, \hat{X}) u(t, q, \dot{q}, e, \dot{e}) .
$$

Let us observe that control law (3.6), (3.7) requires on-line measurements of quantities $q, \dot{q}, e$ and $\dot{e}$, respectively which are assumed for a moment to be available. 
A few remarks may be made regarding the controller (3.6)-(3.7).

- Remark 1. Observe that control law given by Eqs (3.6)-(3.7) is a feed-back adjustable function equal to $\mathcal{V}+\mathcal{W}+c^{\prime}$. The control laws known from the literature (see e.g., Bartolini et al. [46]; Bartolini et al. [47]; Bartolini et al. [48]; Ferrara and Capisani, [49]; Spong and Vidyasagar, [35]; Siciliano, et al. [4] require boundedness of $\dot{q}$ which implies large controller gains to cope with the uncertainty over the whole operation region.

- Remark 2. It is also worth to notice that our feed-back adjustable amplitude term $\frac{c}{a}\left(\mathcal{V}+\mathcal{W}+c^{\prime}\right)$ makes it possible to cope with globally unbounded uncertainties. In general, in that case, only local uncertainty suppression is available in the literature for redundant systems.

- Remark 3. Non-singular sliding variable $s$ defined by Eq.(3.5) has a nice property for a typical regulation task $\dot{e}(0)=0$ which implies $s(0)=0$ and consequently $s(t)=0(\operatorname{TSM} s=0$ is attained at $t=0)$.

- Remark 4. Let us note that expressions Eqs (3.6)-(3.7) present also a transposed Jacobian controller. In such a context, the use of the transpose of the Jacobian is a well-known technique and there are several papers Wolovich and Elliot, [50]; Siciliano, [51]; Kelly, [52]; Cheah, [53]; Cheah, [54]; Moosavian and Papadopoulos, [55] that include its stability analysis. Nevertheless, work Wolovich and Elliot, [50] guarantees only ultimate boundedness provided that $\dot{p}_{d}(t)$ is globally norm bounded. The asymptotically stable purely kinematic control scheme offered in Siciliano, [51] indeed eliminates explicit computation of the inverse but introduces undesirable chattering effect. In work Cheah, [53], it is claimed that controllers based on transposed Jacobian and inverse Jacobian are dual in the sense that the transformation from task space to joint space can be either defined as transposed Jacobian or inverse Jacobian. As was also shown in Cheah, [53]; Cheah, [54], approximate transpose Jacobian control law is asymptotically stable. In work Moosavian and Papadopoulos, [55], a modified transpose Jacobian algorithm was developed which employs stored data of the control command in the previous time step, as a learning tool to yield an improved performance. However, works Kelly, [52]; Cheah, [53]; Cheah, [54]; Moosavian and Papadopoulos, [55] have shown stability of the performance for the set-point control problems. On the other hand, transposed Jacobian controller (3.6)-(3.7) is able to attain the stable equilibrium $(e, \dot{e})=(0,0)$ in a finite time. Due to involving the sliding mode term in $u$, controller (3.6)-(3.7) is also robust against uncertainties of both kinematic and dynamic equations and external unbounded disturbances.

- Remark 5. In controller (3.6)-(3.7), the term $\frac{s}{\|s\|}$ will cause undesirable chattering effect in a small neighbourhood of $s=0$ which results in torque oscillations and consequently in robot vibrations. In order to eliminate the chattering and consequently reduce robot vibrations, a boundary layer control law may be used in place of $u$ given by Eq.(27) of the following form

$$
u(t, q, \dot{q}, e, \dot{e})= \begin{cases}-\frac{c}{a} \frac{s}{\|s\|}\left(\mathcal{V}+\mathcal{W}+c^{\prime}\right) & \text { for }\|s\| \geq \epsilon \\ -\frac{c}{a} \frac{s}{\epsilon}\left(\mathcal{V}+\mathcal{W}+c^{\prime}\right) & \text { otherwise }\end{cases}
$$

here $\epsilon$ is a user specified arbitrarily small positive real number. 
- Remark 6. Let us note that the performance improvement of controller (3.7) is achieved by an increase of control contribution $\mathcal{V}(t, e, \dot{e})$ in Eq.(3.6) for small tracking errors, because for $0<\alpha_{1}<1$ and $\left|e_{i}(t)\right|<1$ we have $\left|e_{i}(t)\right|^{\alpha_{1}}>\left|e_{i}(t)\right|$. Also, when the tracking error is large (especially at the beginning of the control process), the terminal sliding mode controller (3.7) gives smaller control effort than that resulting from a linear sliding mode since $\left|e_{i}(t)\right|^{\alpha_{I}}<\left|e_{i}(t)\right|$ for $\left|e_{i}(t)\right|>1$.

- Remark 7. Let us note that if the conventional trajectory tracking is only taken into account (without objective function $\mathcal{A}$ ) in the control problem then the robotic manipulator becomes strictly redundant, i.e., $n>m$. In such a case by defining the task space TSM $s^{e}$ as $s^{e}=\dot{e}^{e}+\int_{0}^{t}\left(\lambda_{0}\left(e^{e}\right)^{\alpha_{1}}+\lambda_{l}\left(e^{e}\right)^{\alpha_{2}}\right) d \tau$ controller (3.6) and (3.7) may be simplified to the following form

$$
v=\hat{\jmath}^{T} u^{e}\left(t, q, \dot{q}, e^{e}, \dot{e}^{e}\right)
$$

where $\hat{\jmath}=\jmath(q, \hat{X})$ and

$$
u^{e}\left(t, q, \dot{q}, e^{e}, \dot{e}^{e}\right)= \begin{cases}-\frac{c_{e}}{a_{e}} \frac{s^{e}}{\left\|s^{e}\right\|}\left(\mathcal{V}_{e}+\mathcal{W}_{e}+c_{e}^{\prime}\right) & \text { for } s^{e} \neq 0 \\ 0 & \text { otherwise }\end{cases}
$$

$$
\mathcal{V}_{e}=\left\|\lambda_{0}\left(e^{e}\right)^{\alpha_{1}}+\lambda_{1}\left(\dot{e}^{e}\right)^{\alpha_{2}}-\ddot{p}_{d}^{e}\right\| ; \quad \mathcal{W}_{e}=\|J\|_{F} \cdot \Lambda_{\max } \cdot \varepsilon+\left\|\frac{\partial J}{\partial q}\right\|_{F} \cdot\|\dot{q}\|^{2} ; \quad c_{e}>\frac{1}{1-\delta}, c_{e}^{\prime}>0 ; a_{e} \text { denotes }
$$

estimation of the minimal eigenvalue of matrix $\hat{J} M^{-1} \hat{J}^{T}$ along desired trajectory $p_{d}$;

$\|J\|_{F} \leq c_{3} ;\left\|\frac{\partial J}{\partial q}\right\|_{\mathrm{F}} \leq c_{5}$.

In most cases, real robotic manipulators are equipped with encoders which measure only joint positions and/or task errors. Hence, reconstruction or estimation of joint velocity and task space error velocity is required to apply controller (3.6)-(3.7) or (3.7), (3.8). Application of Luenberger-style observers Canudas de Wit et al. [56]; ElBeheiry et al. [57], high-gain observers Khalil and Praly, [58]; Ball and Khalil, [59], model-free observers De Luca et al. [60]; Hsiao and Weng, [61] or a class of observers based on the sliding-mode algorithms Davila et al. [62] seems to be an efficient approach to reconstruct both $\dot{q}$ and $\dot{e}$. Although all the aforementioned observers are able to reliably reconstruct manipulator state (both joint velocity and task space error velocity) based on position measurement $q$ and task error $e$, there appears a difficulty to combine our control law and an observer from Canudas de Wit et al. [56]; ElBeheiry et al. [57]; Khalil and Praly, [58]; Ball and Khalil, [59]; De Luca et al. [60]; Hsiao and Weng, [61]. In order to make such combination possible, observers proposed in works Canudas de Wit et al. [56]; ElBeheiry et al. [57]; Khalil and Praly, [58]; Ball and Khalil, [59]; De Luca et al. [60]; Hsiao and Weng, [61] have to satisfy the so-called separation principle Atasi and Khalil, [63] which implies both the continuity of the controllers from Canudas de Wit et al. [56]; ElBeheiry et al. [57]; Khalil and Praly, [58]; Ball and Khalil, [59]; De Luca et al. [60]; Hsiao and Weng, [61] with the fully available state and asymptotic stability of the closed-loop system under the continuous state feedback controllers. Let us observe that our control law Eqs (3.6)-(3.7) is discontinuous and Eq.(3.7), Eq.(3.8) 
is not asymptotically stable what prevents an application of the state observers from Canudas de Wit et al. [56]; ElBeheiry et al. [57]; Khalil and Praly, [58]; Ball and Khalil, [59]; De Luca et al. [60]; Hsiao and Weng, [61]. Although the observer offered in Davila et al. [62] fulfils the separation principle, our controller handles unbounded uncertainties (in kinematics, dynamics and disturbances) and does not require boundedness of $\dot{q}$ and $\dot{e}$, respectively. A computationally efficient approach based on the uniform robust exact finite-time differentiation has been recently proposed in works Levant and Livne, [64]; Levant, [65] to numerically find derivatives of absolutely continuous functions. The separation principle is trivially fulfilled for differentiators (model-free observers), Levant and Livne, [64]; Levant, [65]. Assuming that position $q=q(t)$ and task error $e=e(t)$ are known (measurable), one can exactly reconstruct both joint velocity $\dot{q}(t)$ and task error velocity $\dot{e}(t)$ (by neglecting the measurement noise of a device) after finite-times of transient processes, say $T_{q}^{\prime}, T_{e}^{\prime}>0$, respectively.

The first-order uniform robust exact differentiators (model-free observers) take in our case the following forms

$$
\begin{aligned}
& \dot{y}_{0}=y_{1}-\hat{\lambda}_{1}^{q} L_{q}(t)^{1 / 2}\left|y_{0}-q\right|^{1 / 2} \operatorname{sign}\left(y_{0}-q\right), \\
& \dot{y}_{1}=-\hat{\lambda}_{0}^{q} L_{q}(t) \operatorname{sign}\left(y_{0}-q\right)
\end{aligned}
$$

and

$$
\begin{aligned}
& \dot{z}_{0}=z_{1}-\hat{\lambda}_{1}^{e} L_{e}(t)^{1 / 2}\left|z_{0}-e\right|^{1 / 2} \operatorname{sign}\left(z_{0}-e\right), \\
& \dot{z}_{1}=-\hat{\lambda}_{0}^{e} L_{e}(t) \operatorname{sign}\left(z_{0}-e\right)
\end{aligned}
$$

where $\hat{\lambda}_{0}^{q}, \hat{\lambda}_{1}^{q}, \hat{\lambda}_{0}^{e}, \hat{\lambda}_{1}^{e}$, are positive constants. $y_{1}, z_{1}$ denote the outputs of differentiators Eqs (3.11)-(3.12) exactly reconstructing joint velocity $\dot{q}(t)$ and task error velocity $\dot{e}(t)$, i.e., $\dot{q}(t)=y_{l}(t), \dot{e}(t)=z_{l}(t)$ for $t \geq \max \left\{T_{q}^{\prime}, T_{e}^{\prime}\right\} \cdot y_{0}, z_{0}$ stand for estimations of $q$ and $e$, respectively. By substituting $\dot{q}$ and $\dot{e}$ in Eq.(3.6) or Eq.(3.8) for their corresponding estimates $y_{1}$ and $z_{1}$ from Eqs (3.11)-(3.12), we obtain the following trajectory tracking controller

$$
v=\hat{J}^{T} u\left(t, q, y_{1}, e, z_{1}\right),
$$

with

$$
u\left(t, q, y_{1}, e, z_{l}\right)= \begin{cases}-\frac{c}{a} \frac{s}{\|s\|}\left(\mathcal{V}+\mathcal{W}+c^{\prime}\right) & \text { for }\|s\| \geq \epsilon, \\ -\frac{c}{a} \frac{s}{\epsilon}\left(\mathcal{V}+\mathcal{W}+c^{\prime}\right) & \text { otherwise, }\end{cases}
$$

which requires only the measurements of joint position $q$ and task error $e . L_{q}(t), L_{e}(t)$ stand for positive continuous functions which take the forms $L_{q}(t)=\Lambda_{\max }(\|v\|+\varepsilon)$, and $L_{e}(t)=\|J\|_{F} \cdot L_{q}(t)+\left\|\frac{\partial J}{\partial q}\right\|_{F} \cdot\left\|y_{1}\right\|^{2}+\left\|\ddot{p}_{d}\right\|$, respectively. $L_{q}(t), L_{e}(t)$ represent physically upper estimations of the norms of $\ddot{q}, \ddot{e}$ (manipulator joint acceleration and task error acceleration), respectively. In a general case, if measured joint position $q=q(t)$ 
and task error $e=e(t)$, obtained from encoders, are additionally contaminated by a measurement noise $\eta(t)$, i.e., $q(t)=q_{0}(t)+\eta(t), \quad e(t)=e_{0}(t)+\eta(t)$, where $\|\eta\| \leq \rho \cdot \min \left\{L_{q}(t), L_{e}(t)\right\} ; \rho$ denotes a normalised noise magnitude (practically $\rho \in\left[10^{-4}, 10^{-3}\right]$ ); $q_{0}(t), \dot{q}_{0}(t), e_{0}(t), \dot{e}_{0}(t)$ stand for unknown true (noisefree) joint position, velocity, task error and task error velocity, respectively, then observers (3.11), (3.12) should also be applied to estimate quantities $e$ and $\dot{e}$.

\section{Computer example}

This section demonstrates the performance of the controllers given by expressions (3.6)-(3.7); (3.7), (3.8); (3.9)-(3.10) and (3.13)-(3.14) on a selected robotic manipulator task. Moreover, a numerical comparison of our control schemes to other well-known controllers is also drawn. For this purpose, we utilise a dynamic three-joint direct-drive arm $(n=3)$ of a SCARA-type robotic manipulator operating in the two-dimensional task space $(m=2)$ whose kinematic scheme is shown in Fig.1, where $X=\left(X_{1}, X_{2}, X_{3}\right)^{T}$ stands for the ordered set of link lengths.

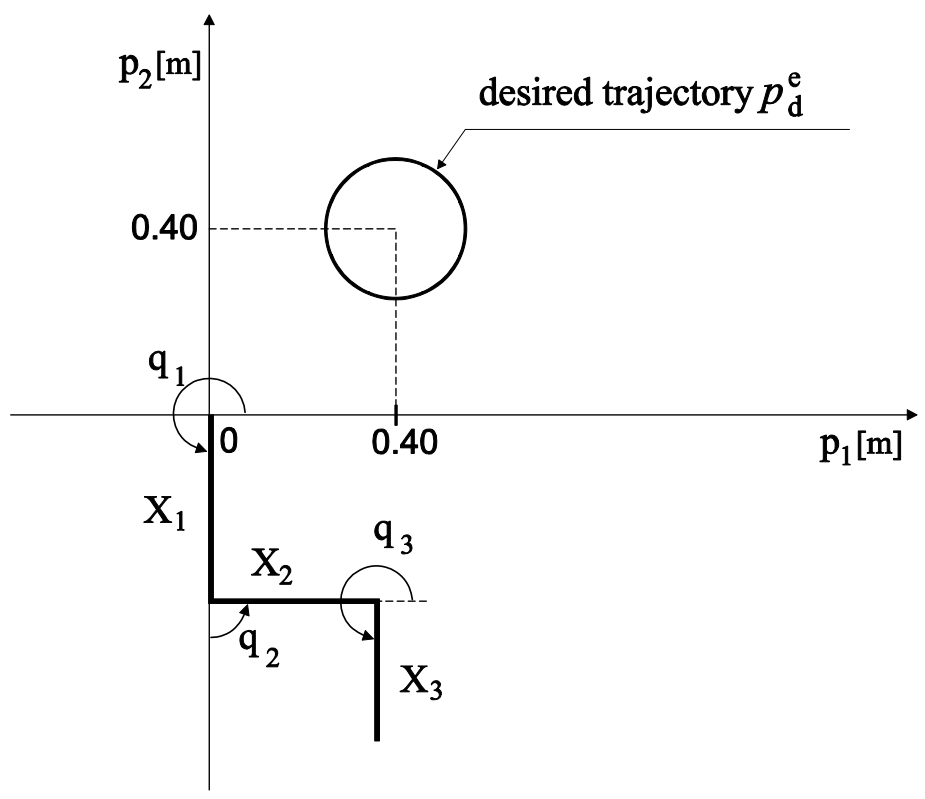

Fig.1. A kinematic scheme of the manipulator and the task to be accomplished.

In all numerical simulations, the SI units are used. The (nominal) link lengths are assumed to be equal $X_{n}=(0.40 .360 .3)^{T}$. The estimation term $\hat{X}$ takes in all the simulations the form as follows $\hat{X}=\left(\begin{array}{lll}0.5 & 0.28 & 0.35\end{array}\right)^{T}$. The coefficients of disturbance term $D$ and their estimations are assumed in all the experiments to take the following scalar values: $d_{0}=\bar{d}_{0}=2, d_{1}=\bar{d}_{1}=2, d_{2}=5, \bar{d}_{2}=7, d_{3}=2, \bar{d}_{3}=3, d_{4}=0.2$ and $\underline{d}_{4}=0$, respectively. In order to numerically compare our controllers with those known from the literature, we reformulate dynamic equations (2.1), (2.12) to a partially linearly parameterizable form as follows Spong and Vidyasagar, [35]

$$
M \dot{r}+C r+\Theta\left(e^{e}, \dot{e}^{e}, q, \dot{q}, \mathcal{A}, \dot{\mathcal{A}}\right) Y+d_{2} \operatorname{sign}(\dot{q})+d_{3} \exp \left(-d_{4}\|\dot{q}\|^{2}\right) \operatorname{sign}(\dot{q})=v
$$


where $r=\dot{q}-J^{\#}\left(\dot{p}_{d}-\alpha e^{e}\right)-\left(\mathbb{I}_{n}-\jmath^{\#} J\right) 10 \frac{\partial \mathcal{A}}{\partial q}$, denotes a filtered tracking error signal Zergeroglu et al. [22]; Ozbay et al. [23] $J=J\left(q, X_{n}\right) ; \quad \Theta=\Theta\left(e^{e}, \dot{e}^{e}, q, \dot{q}, \mathcal{A}, \dot{\mathcal{A}}\right) \in \mathbb{R}^{n \times d}, \quad$ is the regression matrix; $\Theta Y=M \frac{d}{d t}\left(J^{\#}\left(\dot{p}_{d}-\alpha e^{e}\right)+\left(\mathbb{I}_{n}-J^{\#} J\right) 10 \frac{\partial \mathcal{A}}{\partial q}\right)+C\left(J^{\#}\left(\dot{p}_{d}-\alpha e^{e}\right)+\left(\mathbb{I}_{n}-J^{\#} J\right) 10 \frac{\partial \mathcal{A}}{\partial q}\right)+G+d_{0}(q-q(0))+$

$d_{1} \dot{q} ; d$ stands for the minimal number of the dynamic parameters, which are combinations of physical link parameters of individual links and coefficients of continuous linear friction terms; $Y$ is the constant parameters vector (link mass, link inertia, friction coefficients, etc.); $\alpha$ stands for a constant gain coefficient. The components of the dynamic equations of the manipulator from Fig.1, (without disturbance term $D$ ) are as follows Galicki, [20]

$$
\begin{aligned}
& M=\left[M_{i j}\right]_{1 \leq i, j \leq 3}, \\
& M_{11}=Y_{1}+2 Y_{4} c 2+Y_{5} c 23+Y_{6} c 3, \\
& M_{12}=Y_{2}+Y_{4} c 2+\frac{Y_{5}}{2} c 23+Y_{6} c 3, \\
& M_{13}=Y_{3}+\frac{Y_{5}}{2} c 23+\frac{Y_{6}}{2} c 3, \\
& M_{21}=M_{12}, \\
& M_{22}=Y_{2}+Y_{6} c 3, \\
& M_{23}=Y_{3}+\frac{Y_{6}}{2} c 3, \\
& M_{31}=M_{13}, \\
& M_{32}=M_{23}, \\
& M_{33}=Y_{3}, \\
& C_{12}=-\left(C_{i j}\right]_{1 \leq i, j \leq 3}, \\
& C_{11}=-\left(Y_{4} s 2+Y_{5} s 12\right) \dot{q}_{2}-\left(Y_{5} s 12+Y_{6} s 12\right) \dot{q}_{3}, \\
& \left.C_{5} s 12\right)\left(\dot{q}_{1}+\dot{q}_{2}\right)-\left(Y_{5} s 12+Y_{6} s 12\right) \dot{q}_{3}, \\
& \left.C_{6}+Y_{6} s 12\right)\left(-\dot{q}_{1}+\dot{q}_{2}+\dot{q}_{3}\right), \\
& C_{13},
\end{aligned}
$$




$$
\begin{aligned}
& C_{21}=\left(Y_{4} s 2+Y_{5} s 12\right) \dot{q}_{1}+Y_{6} s 3 \dot{q}_{3}, \\
& C_{22}=-\left(Y_{5} s 12+Y_{6} s 12\right) \dot{q}_{3}, \\
& C_{23}=-Y_{6} s 3\left(3 \dot{q}_{1}+\dot{q}_{2}+\dot{q}_{3}\right), \\
& C_{31}=\left(Y_{4} s 2+Y_{5} s 12\right) \dot{q}_{1}-Y_{6} s 3 \dot{q}_{2}, \\
& C_{32}=Y_{6} s 3\left(\dot{q}_{1}+\dot{q}_{2}\right), \\
& C_{33}=0, \\
& G=\left(\begin{array}{c}
Y_{7} c 1+Y_{8} c 12+Y_{9} c 123 \\
Y_{8} c 12+Y_{9} c 123 \\
Y_{9} c 123
\end{array}\right)
\end{aligned}
$$

where $\quad c i=\cos \left(q_{i}\right) ; \quad s i=\sin \left(q_{i}\right) ; \quad c i j=\cos \left(q_{i}+q_{j}\right) ; \quad s i j=\sin \left(q_{i}+q_{j}\right) ; \quad c i j k=\cos \left(q_{i}+q_{j}+q_{k}\right)$; sijk $=\sin \left(q_{i}+q_{j}+q_{k}\right)$. Parameters $Y=\left(Y_{1}, \ldots, Y_{9}, d_{0}, d_{1}\right)^{T}$ (including coefficients of continuous linear friction terms) take the following nominal values: $Y_{n}=(7.7,2.4,0.4,2.6,1.3,1.2,128,64,16,2,2)^{T}$. Based on Eqs (4.1)-(4.4) and the knowledge of $X_{n}, Y_{n}$, a numerical comparison of our controller (3.13)(3.14) to a well-known robust controller from Zergeroglu et al. [22]; Ozbay et al. [23] will also be carried out in this section. The control law proposed in Zergeroglu et al. [22]; Ozbay et al. [23] is given by the following expression

$$
v=\Theta \hat{Y}+K r-J^{T} e^{e}+v_{R}
$$

where $v_{R}=\frac{r \Delta^{2}}{\|r\| \Delta+\epsilon}$ is an auxiliary robust control term designed to compensate for unknown nonlinear part of disturbances and parameter uncertainties of terms $M, C$ and $G$, respectively; $K$ denotes a gain coefficient, $\hat{Y}$ means the best guess estimates (of the unknown parameters $Y$ ). Let us note that controller (4.5) requires the full knowledge of kinematic parameters $X$. Moreover, according to Zergeroglu et al. [22]; Ozbay et al. [23], the best values for $\Delta$ and $\epsilon$ equal $\Delta=10, \epsilon=0.01$, respectively. The initial configuration and velocity of the manipulator are equal in all the experiments to $q(0)=\left(-\frac{\pi}{2}, \frac{\pi}{2}, 0\right)^{T}, \dot{q}(0)=(0,0,0)^{T}$, respectively.

To compare the performance of controllers (3.6)-(3.7) and (3.9)-(3.10), our estimates for controllers (3.6)-(3.7); (3.9)-(3.10); (3.7), (3.8) and (3.13)-(3.14) are chosen as $a=a_{e}=0.2 ; \Lambda_{\max }=6$. In order to simplify numerical computations, rough conservative estimations of $c_{i}, i=1: 6$ have been assumed. Hence, positive constant coefficients $c_{i}, i=1: 6$ were chosen as follows $c_{1}=10, c_{2}=240, c_{3}=0.07$, $c_{4}=0.0007, c_{5}=0.00022$ and $c_{6}=0.000022$, respectively. 
The task realized by controllers (3.6)-(3.7); (3.9)-(3.10); (3.7), (3.8); (3.13)-(3.14) and (4.5), is to track by the end-effector a circle trajectory, expressed by the following equations (see the solid circle trajectory in Fig.1)

$$
p_{d}^{e}(t)=(0.4+0.15 \cos (t), 0.4+0.15 \sin (t))^{T}
$$

In order to attain the convergence of task errors $e^{e}$ less or equal to $10^{-3}$ in approximately the same time, the following numerical values of gain coefficients for all the controllers are taken: $K=245, \alpha=374.5$, $K_{\mathcal{A}}=0.05, \quad c_{\mathcal{A}}=1, \quad q_{\text {rest }}=q(0), \quad c=c_{e}=1.46, \quad c^{\prime}=c^{\prime}{ }_{e}=0.5, \quad \lambda_{0}=51, \quad \lambda_{1}=46, \quad$ and $\quad \alpha_{1}=\frac{3}{5}$, respectively. In order to speed up the convergence process of differentiators (3.11)-(3.12), we have chosen good initial guesses $y_{1}(0), z_{l}(0)$ in the numerical examples (which imply relations $T_{q}^{\prime} \simeq 0$ and $T_{e}^{\prime} \simeq 0$ ) based on the nominal values of both our kinematic and dynamic models. Consequently, differentiators (3.11)-(3.12) were run with the following initial values $y_{0}=q(0), y_{1}(0)=\dot{q}(0), z_{0}(0)=e(0)=(0.11-0.80)^{T}$, $z_{l}(0)=\dot{e}(0)=(0-0.150)^{T}$ and parameters $\hat{\lambda}_{0}^{q}=\hat{\lambda}_{0}^{e}=1.1 ; \hat{\lambda}_{1}^{q}=\hat{\lambda}_{1}^{e}=1.4$, respectively. Due to conservative nature of estimates $L_{q}$ and $L_{e}$ in Eqs (3.11)-(3.12), they are assumed for simplicity of computations in all the simulations to be equal to $L_{e}(t)=L_{q}(t)=\Lambda_{\max }(\|v\|+\varepsilon)$. Let us also observe that filtered tracking error $r$ in Eq.(4.5) plays a role of linear sliding variable with respect to $e^{e}$ whereas its corresponding term $s$ in Eqs (3.13)-(3.14) is a non-linear function of $e^{e}$. In order to better visualize time courses of the task errors for all the controllers, we omit in the simulations an initial approaching phase of the end-effector to desired trajectory $p_{d}^{e}$. Consequently, the trajectory tracking is exhibited for $t \in[5,9]$.

The aim of the first experiment is to both compare the performance of the two proposed controllers (3.6)-(3.7); (3.9)-(3.10) and exhibit the role of objective function $\mathcal{A}$ by reducing the torque $v$ in the $L_{2}[1,9]$ norm. The results of the simulations are depicted in Figs 2-6. As is seen from Figs 2, 4, both controllers generate tracking errors $e^{e}$ which are practically equal to zero. Moreover, Fig.5 indicates that controller (3.6)-(3.7) provides optimal manipulator movements ( $e^{a}$ practically equals 0$)$ whereas control law (3.9)-(3.10) does not generate optimal solution $\left(e^{a} \neq 0\right)$. As is also seen from Fig.3, Fig.6, control scheme (3.6)-(3.7) results in smaller torque norm $\overline{\|v\|}=389$ (defined in $L_{2}[1,9]$ norm as $\overline{\|v\|}=\frac{1}{2} \int_{1}^{9} \sqrt{\langle v, v\rangle d t \text { ) }}$ than that obtained from Eqs (3.9)-(3.10) and equal to $\overline{\|v\|}=611$.

In order to both eliminate the chattering effect and exhibit the role of feed-back amplitude adjustable term $\frac{c}{a}\left(\mathcal{V}+\mathcal{W}+c^{\prime}\right)$ from Eqs (3.13)-(3.14) compared with the corresponding constant term $\Delta$ from Eq.(4.5) in the second experiment, components of dynamic equations Eqs (4.2)-(4.4) together with coefficients of continuous linear friction terms $d_{0}, d_{1}$ are assumed in this experiment to be fully known, i.e., the following equality is now fulfilled: $\hat{Y}=Y_{n}$. Moreover, application of controller (4.5) requires the full knowledge of the set $X$, i.e., we assume that $X=X_{n}$. The results of computer simulations are presented in Figs 7-9. As is seen from Figs 7-8, controller (3.13)-(3.14) generates tracking errors $e^{e}$ and $e^{a}$ which are also practically equal to zero compared with controller (4.5) using the full knowledge of both robot kinematic and dynamic equations. This fact is a consequence of linear dependence of $r$ on $e^{e}$. 


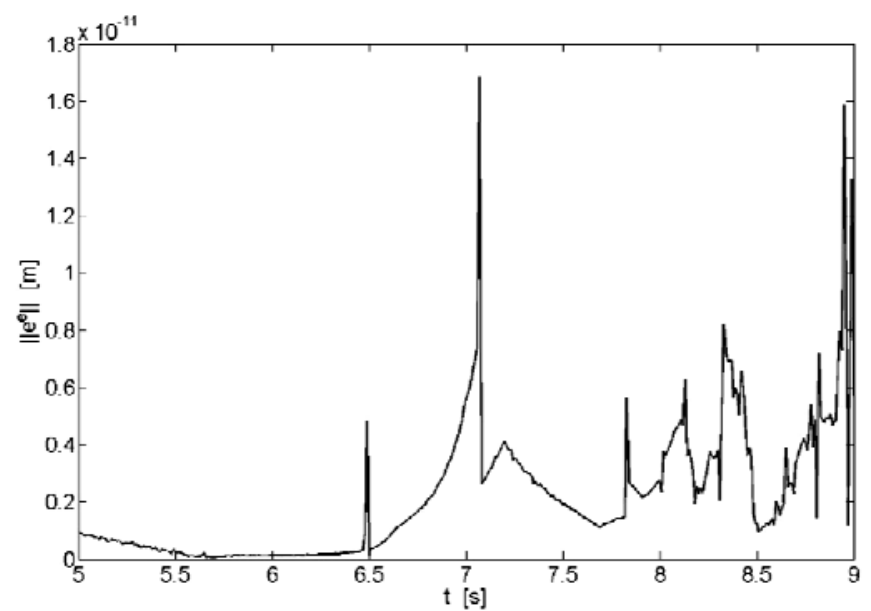

Fig.2. Position errors $e^{e}$ for controller (3.9)-(3.10) when tracking the trajectory $p_{d}$.

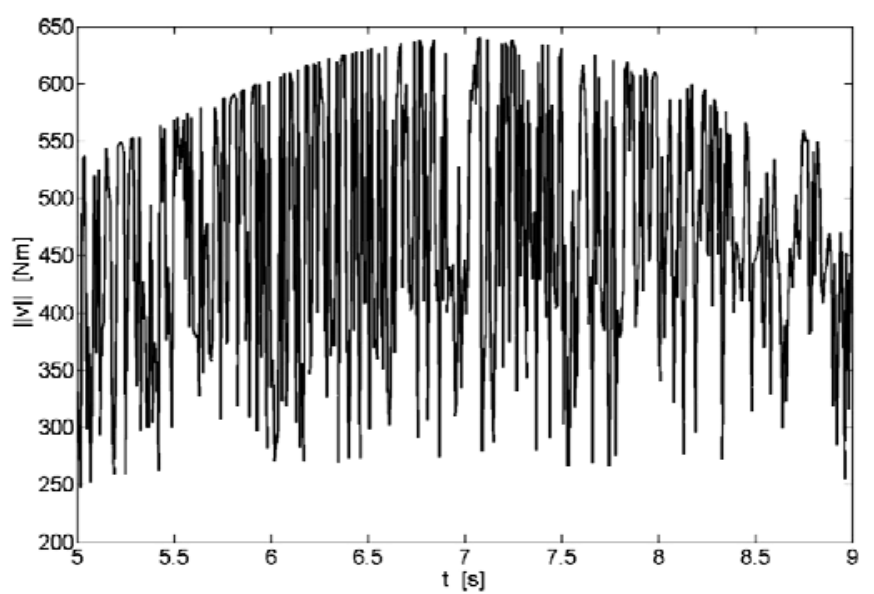

Fig.3. Euclidean norm of $v$ for controller (3.9)-(3.10) when tracking the trajectory $p_{d}$.

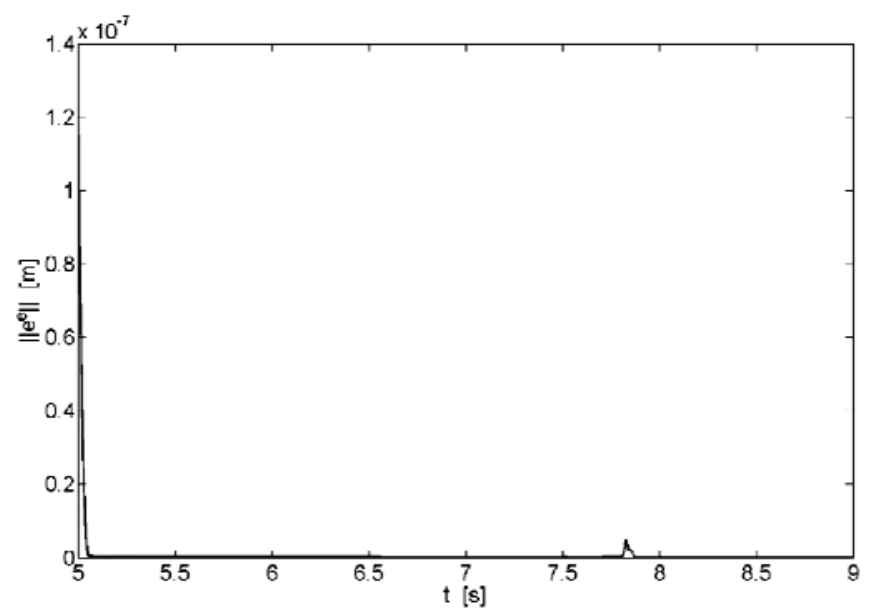

Fig.4. Position errors $e^{e}$ for controller (3.6)-(3.7) when tracking the trajectory $p_{d}$. 


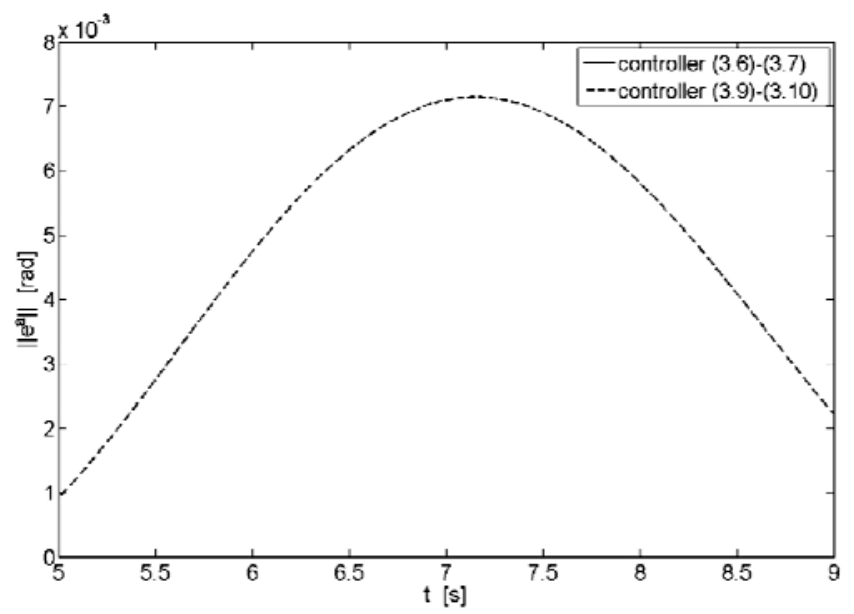

Fig.5. Task errors $e^{a}$ for controllers (3.6)-(3.7) and (3.9)-(3.10) when tracking the trajectory $p_{d}$.

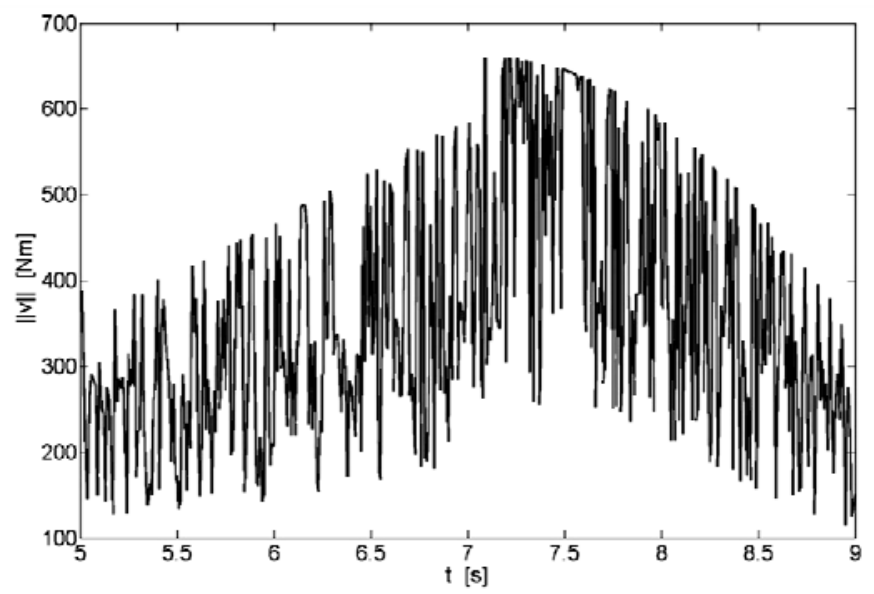

Fig.6. Euclidean norm of $v$ for controller (3.6)-(3.7) when tracking the trajectory $p_{d}$.

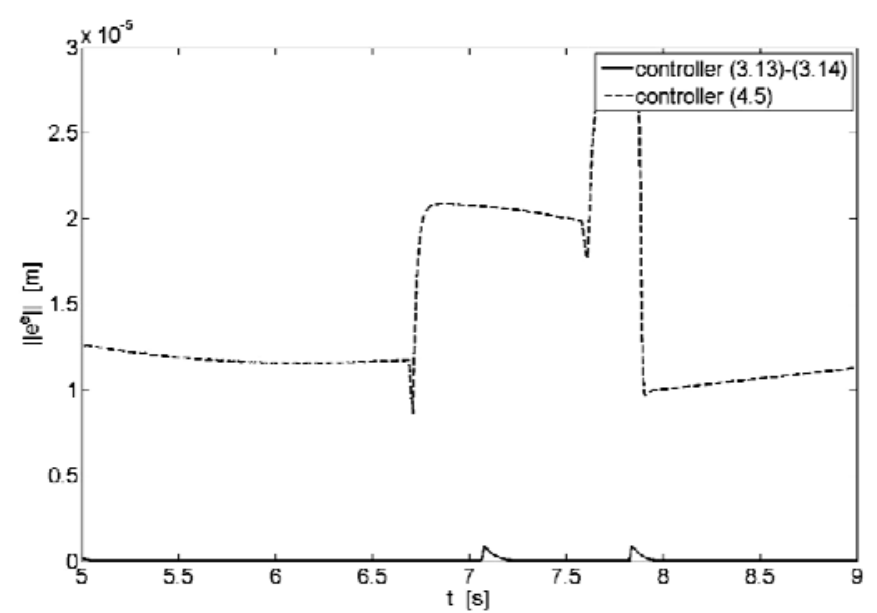

Fig.7. Position errors $e^{e}$ for controllers (3.13)-(3.14) and (4.5) when tracking the trajectory $p_{d}$. 
Moreover, Fig. 8 indicates that controller (3.13)-(3.14) provides practically optimal manipulator movements whereas control law (4.5) generates only suboptimal solution (controller (4.5) results in $e^{a} \neq 0$ ). As is also seen from Fig.9, control scheme (3.13)-(3.14) results in smaller torque norm $\overline{\|v\|}=184.7$ than that obtained from Eq.(4.5) and equal to $\overline{\|v\|}=202.8$. This fact is a consequence of both torque minimizing role of objective function $\mathcal{A}$ and Remarks 1, 2 explaining the role of the feed-back adjustable amplitude term $\frac{c}{a}\left(\mathcal{V}+\mathcal{W}+c^{\prime}\right)$ which varies over the manipulator operation region with respect to the changing uncertainty bounds. Alternatively, the corresponding amplitude term $\Delta$ from Eq.(4.5) is constant in the whole operation region.

In the third experiment, both measured position $q$ and task error $e$ have additionally been contaminated by a measurement noise of a Brownian motion of the form $d \eta(t)=10^{-3} \sqrt{t} X(t) d t$ for $t \in[0,9]$; $X(t) \sim N(0,1)$. The results of simulations are given in Figs 10-11, which indicate a good performance of controller (3.13)-(3.14) subject to measurement noise. The peak of $\|\hat{e}\|$ for $t \simeq 7.2$ in Fig.10, is a result of the Coulomb and Stribeck discontinuity friction terms with respect to $\dot{q}$ depicted in Fig.11.

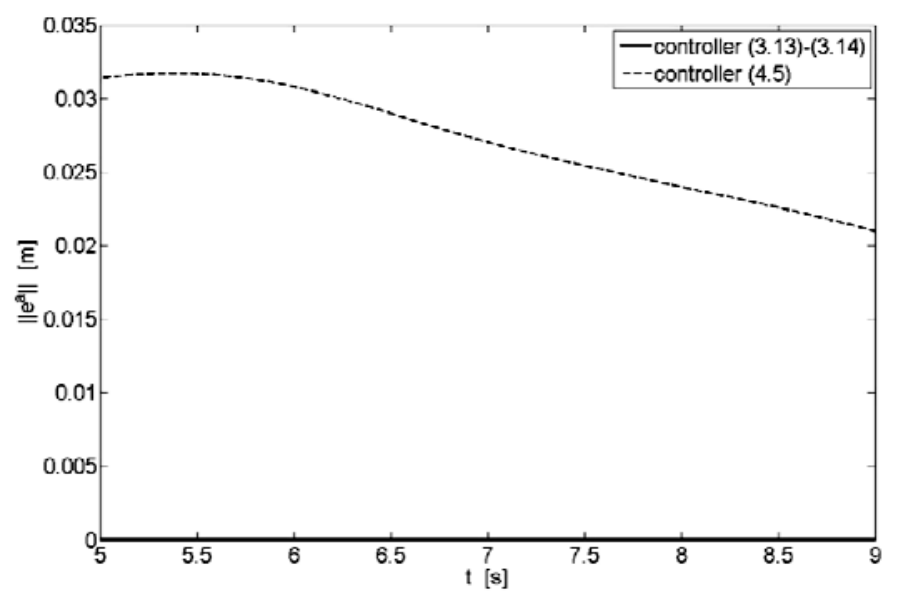

Fig.8. Task errors $e^{a}$ for controllers (3.13)-(3.14) and (4.5) when tracking the trajectory $p_{d}$.

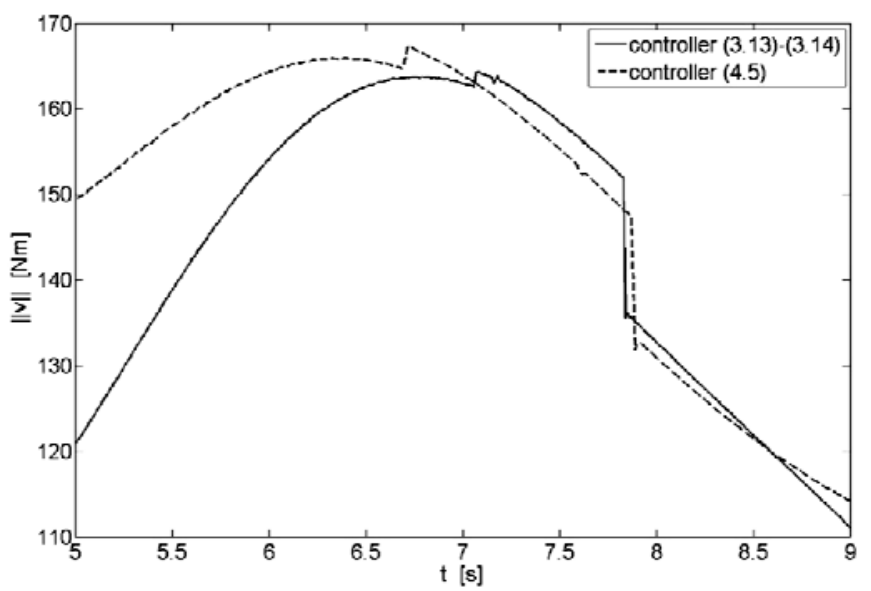

Fig.9. Euclidean norm of $v$ for controllers (3.13)-(3.14) and (4.5) when tracking the trajectory $p_{d}$. 


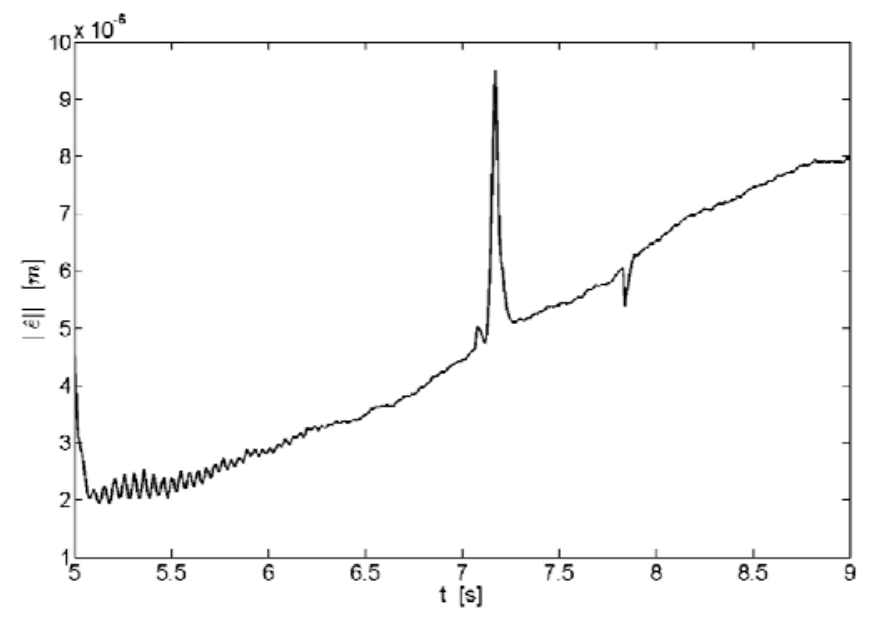

Fig.10. Euclidean norm of estimated task errors $\hat{e}$ for controller (3.13)-(3.14) with measurement noise.

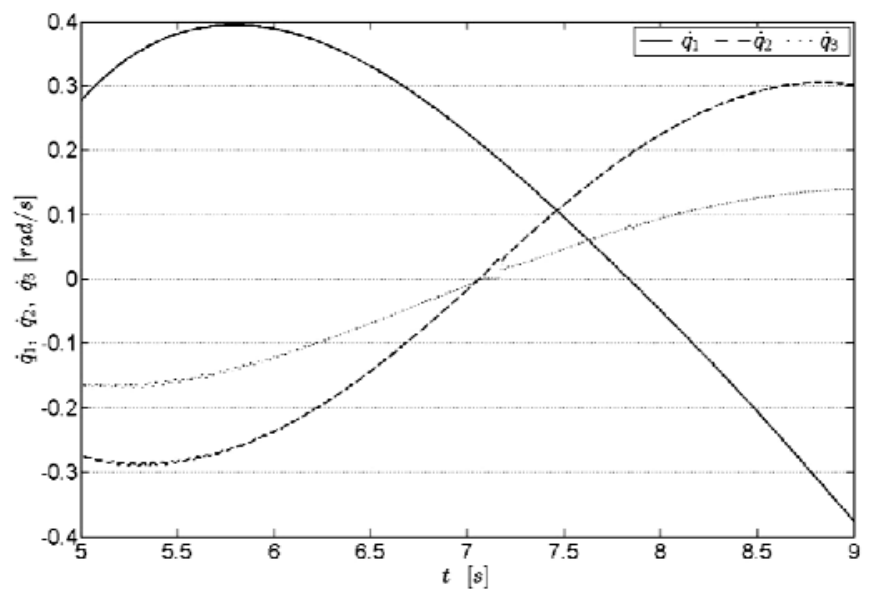

Fig.11. Joint velocities $\dot{q}$ for controller (3.13)-(3.14) with measurement noise.

\section{Conclusions}

A new class of task space TSM controllers with the finite-time convergence property of the desired trajectory tracking by $n$-DoF redundant manipulator whose end-effector operates in the $m$-dimensional task space, has been proposed in this paper. Moreover, a novel non-singular TSM manifold was incorporated into control scheme. The main feature of the control law proposed is both the elimination of the manipulator Jacobian inverse or pseudo-inverse from the end-effector trajectory tracking and copying with the unbounded disturbances. Instead, estimate of the Jacobian transpose matrix has been used. In order to eliminate undesirable chattering effect, a boundary layer control was proposed guaranteeing arbitrarily small bounds on the tracking errors without increase of the controller gains. Numerical simulations have shown that controller (3.13)-(3.14) is superior to a well known robust control scheme (4.5) in accuracy of the trajectory tracking. Although our control technique needs knowledge extracted from the system equations of the robot, the approach is able to handle uncertainty (in kinematics, dynamics and disturbance) occurring in the system. 


\section{Nomenclature}

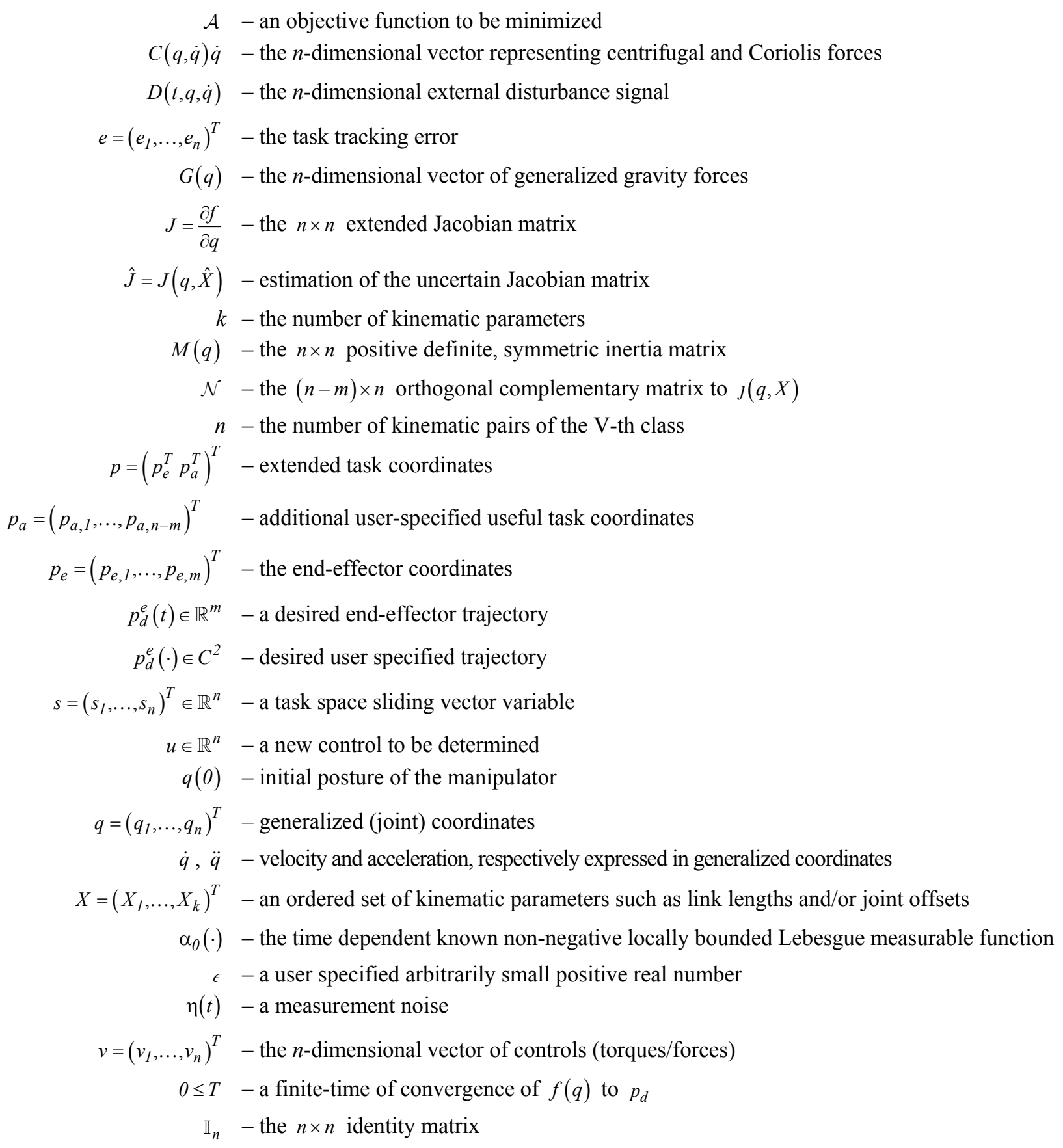

\section{References}

[1] Khatib O. (1987): A unified approach for motion and force control of robot manipulators. - EEE Journal on Robotics and Automation, vol.3, No.1, pp.43-53.

[2] Hsu P., Hauser J. and Sastry S. (1989): Dynamic control of redundant manipulators. - Journal of Robotic Systems, vol.6, No.2, pp.133-148.

[3] Canudas C., Siciliano B. and Bastin G. (Eds. ) (1996): Theory of Robot Control. - London: Springer. 
[4] Siciliano B., Sciavicco L., Villani L. and Oriolo G. (Eds.) (2010): Robotics: Modelling, Planning and Control. Springer Verlag.

[5] Galicki M. (2004): Path following by the end-effector of a redundant manipulator operating in a dynamic environment. - IEEE Transactions on Robotics, vol.20, No.6, pp.1018-1025.

[6] Kelly R. and Moreno J. (2005): Manipulator motion control in operational space using joint velocity inner loop. Automatica, vol.41, No.8, pp.1423-1432.

[7] Nakanishi J., Cory R., Mistry M., Peters J. and Schaal S. (2008): Operational space control: A theoretical and empirical comparison. - The International Journal of Robotics Research, vol.27, No.6, pp.737-757.

[8] Moreno-Valenzuela J. and Gonzales-Hernandez L. (2011): Operational space trajectory tracking control of robot manipulators endowed with a primary controller of synthetic joint velocity. - ISA Transactions, vol.50, No.1, pp.131-140.

[9] Tatlicioglu E., Braganza D., Burg T.C. and Dawson D.M. (2008): Adaptive control of redundant robot manipulators with sub-task objectives. - In Proc. ACC, pp.856-860.

[10] Sadeghian H., Keshmiri M., Villani L. and Siciliano B. (2012): Priority oriented adaptive control of kinematically redundant manipulators. - In Proc. IEEE RA, pp.293-298.

[11] Sadeghian H., Villani L., Kesmiri M. and Siciliano B. (2013): Dynamic multi-priority control in redundant robotic systems. - Robotica, vol.31, No.07, pp.1155-1167.

[12] Feng, G. and Palaniswami M. (1993): Adaptive control of robot manipulators in task space. - IEEE Trans. Automat. Contr., vol.38, No.1, pp.100-104.

[13] Zergeroglu E., Dawson D.M., Walker I. and Behal A. (2000): Nonlinear tracking control of kinematically redundant robot manipulators. - In Proc. ACC, vol.4, pp.2513-2517.

[14] Braganza D., Dixon W.E., Dawson D.M. and Xian B. (2005): Tracking control for robot manipulators with kinematic and dynamic uncertainty. - In Proc. CDC, pp.5293-5297.

[15] Braganza D., Dixon W.E., Dawson D.M. and Xian B. (2008): Tracking control for robot manipulators with kinematic and dynamic uncertainty. - International Journal of Robotics and Automation, vol.23, No.2, pp.117-126

[16] Galicki M. (2007): Adaptive path-constrained control of a robotic manipulator in a task space. - Robotica, vol.25, No.1, pp.103-112.

[17] Cheah C.C., Liu C. and Slotine J.J. (2006): Adaptive tracking control for robots with unknown kinematic and dynamic properties. - The International Journal of Robotics Research, vol.25, No.3, pp.283-296.

[18] Li X. and Cheah C.C. (2012): Adaptive regional feedback control of robotic manipulator with uncertain kinematics and depth information. - In Proc. ACC, pp.5472-5477.

[19] Li, X. and Cheah C.C. (2013): Global task-space adaptive control of robot. - Automatica, vol.49, No.1, pp.58-69.

[20] Galicki M. (2013): Inverse-free control of a robotic manipulator in a task space. - Robotics and Autonomous Systems, vol.62, No.2, pp.131-141.

[21] Zuo Z. (2013): Adaptive trajectory tracking control design with command filtered compensation for a quadrotor.Journal of Vibration and Control, vol.19, No.1, pp.94-108.

[22] Zergeroglu E., Sahin H.T., Ozbay U. and Tektas H.A. (2006): Robust tracking control of kinematically redundant robot manipulators subject to multiple self-motion criteria. - In Proc. IEEE Control Appl., pp.2860-2865.

[23] Ozbay U., Sahin H.T. and Zergeroglu E. (2008): Robust tracking control of kinematically redundant robot manipulators subject to multiple self-motion criteria. - Robotica, vol.26, No.06, pp.711-728.

[24] Singh H.P. and Sukavanam N. (2012): Neural network based control scheme for redundant robot manipulators subject to multiple self-motion criteria. - Mathematical and Computer Modelling, vol.55, No.3, pp.1275-1300.

[25] Nandhakumar S., Muthukumaran V., Prakash, K.S. and Shunmughanaathan V.K. (2015): Position control of industrial robotic manipulator using variable structure control system with single term Haar wavelet series method. - Journal of Vibration and Control, vol.21, No.12, pp.2465-2483. 
[26] Green A. Sasiadek J.Z. (2004): Dynamics and trajectory tracking control of a two-link robot manipulator. - Journal of Vibration and Control, vol.10, No.10, pp.1415-1440.

[27] Seraji H. and Colbaugh R. (1990): Improved configuration control for redundant robots. - J. Robot. Syst., vol.7, No.6, pp.897-928.

[28] Peng Z.X. and Adachi N. (1993): Compliant motion control of kinematically redundant manipulators. - IEEE Trans. Robot. Automat., vol.9, No.6, pp.831-836.

[29] Ott C., Dietrich A. and Schaffer A.A. (2015): Prioritized multi-task compliance control of redundant manipulators. Automatica, vol.53, pp.416-423.

[30] Oh Y. and Chung W.K. (1999): Disturbance observer based motion control of redundant manipulators using inertially decoupled dynamics. - IEEE/ASME Trans. Mechatronics, vol.4, No.2, pp.133-146.

[31] Colbaugh R. and Glass K. (1995): Robust adaptive control of redundant manipulators. - J. Intell. Robot. Syst., vol.14, No.1, pp.69-88.

[32] Balleieul J. (1985): Kinematic programming alternatives for redundant manipulators. - In Proc. IEEE Int. Conf. on Robotics and Automation, vol.2, pp.722-728.

[33] Shamir T. and Yomdin Y. (1988): Repeatability of redundant manipulators: Mathematical solution of the problem. IEEE Trans. Automat. Cont., vol.33, No.11, pp.1004-1009.

[34] Roberts R.G. and Maciejewski A.A. (1992): Nearest optimal repeatable control strategies for kinematically redundant manipulators. - IEEE Trans. Robot. Automat. vol.8, No.3, pp.327-337.

[35] Spong M.W. and Vidyasagar M. (1989): Robot Dynamics and Control. - New York: Wiley.

[36] Haessing D. and Friedland B. (1991): On the modeling and simulation of friction. - Transactions of the ASME, Journal of Dynamic Systems, Measurements and Control, vol.113, No.3, pp.354-362.

[37] Wit C., Ollson H., Astrom K. and Lischinsky P. (1995): A new model for control of systems with friction. - IEEE Trans. Automat. Contr., vol.40, No.3, pp.419-425.

[38] Yoshikawa T. (1985): Manipulability of robotic mechanisms. - Int. J. Robotics Res., vol.4, No.2, pp.3-9.

[39] Maciejewski, A.A. and Klein C.A. (1985): Obstacle avoidance for kinematically redundant manipulators in dynamically varying environments. - I. J. Rob. Res., vol.4, No.3, pp.109-117.

[40] Perdereau V., Passi, C. and Drouin, M. (2002): Real-time control of redundant robotic manipulators for mobile obstacle avoidance. - Robotics and Autonomous Systems, vol.41, No.1, pp.41-59.

[41] Cruse H. et al. (1990): On the cost functions for the control of the human arm movement. - Biological Cybernetics, vol.62, No.6, pp.519-528.

[42] Feng Y., Yu, X., and Man Z. (2002): Non-singular terminal sliding mode control of rigid manipulators. - Automatica, vol.38, No.12, pp.2159-2167.

[43] Yu S., Yu X., Shirinzadeh B. and Man Z. (2005): Continuous finite-time control for robotic manipulators with terminal sliding mode. - Automatica, vol.41, No.11, pp.1957-1964.

[44] Zhao D., Li S. and Gao F. (2009): A new terminal sliding mode control for robotic manipulators. - International Journal of Control, vol.82, No.10, pp.1804-1813.

[45] Galicki M. (2016): Constraint finite-time control of redundant manipulators. - Int. J. Robust. Nonlinear Control, http://dx.doi.org/10.1002/rnc.3591.

[46] Bartolini G., Ferrara A. and Punta E. (2000): Multi-input second-order sliding-mode hybrid control of constrained manipulators. - Dynamics and Control, vol.10, No.3, pp.277-296.

[47] Bartolini G., Ferrara A., Usai E. and Utkin V.I. (2000): On multi-input chattering-free second-order sliding mode control. - IEEE Transactions on Automatic Control, vol.45, No.9, pp.1711-1717.

[48] Bartolini G., Pisano A., Punta E. and Usai E. (2003): A survey of applications of second-order sliding mode control to mechanical systems. - International Journal of Control, vol.76, No.9-10, pp.875-892. 
[49] Ferrara A. and Capisani L.M. (2011): Second order sliding modes to control and supervise industrial robot manipulators. - In. L. Fridman et al. (Eds.): Sliding modes, LNCIS, Lecture Notes in Control and Information Sciences, vol.412, pp.541-567.

[50] Wolovich W.A. and Elliot H. (1984): A computational technique for inverse kinematics. - In Proc. 23rd IEEE Conference on Decision and Control, pp.1359-1363.

[51] Siciliano B. (1990): A closed-loop inverse kinematic scheme for on-line joint-based robot control. - Robotica, vol.8, No.3, pp.231-243.

[52] Kelly R. (1996): Robust asymptotically stable visual servoing of planar robots. - IEEE trans. Rob. Automat., vol.12, No.5, pp.759-766.

[53] Cheah C.C. (2006): On duality of inverse Jacobian and transpose Jacobian in task-space regulation of robots. In Proc. IEEE Int. Conf. on Robotics and Automation, pp.2571-2576.

[54] Cheah C.C., Lee K., Kawamura S. and Arimoto S. (2000): Asymptotic stability control with approximate Jacobian matrix and its application to visual servoing. - In Proc. IEEE Decision and Control, pp.3939-3944.

[55] Moosavian S.A.A. and Papadopoulos E. (2007): Modified transpose Jacobian control of robotic systems. Automatica, vol.43, No.7, pp.1226-1233.

[56] Canudas de Wit C., Fixot N. and Astrom K.J. (1992): Trajectory tracking in robot manipulators via nonlinear estimated state feedback. - IEEE Trans. Robot. Automat., vol.8, No.1, pp.138-144.

[57] ElBeheiry E.M., Zaki A. and ElMaraghy W.H. (2003): A unified approach for independent manipulator joint acceleration control and observation. - ASME Dynamic Systems and Control Division, vol.72, No.1, pp.659666.

[58] Khalil H.K. and Praly L. (2014): High-gain observers in nonlinear feedback control. - Int. J. Robust and Nonlinear Control, vol.24, No.6, pp.993-1015.

[59] Ball A.A. and Khalil H.K. (2013): A nonlinear high-gain observer for systems with measurement noise. - IEEE Trans. Automat. Control, vol.58, pp.569-580.

[60] De Luca A., Schroder D. and Thummel M. (2007): An acceleration-based state observer for robot manipulators with elastic joints. - In Proc. IEEE International Conference on Robotics and Automation, pp.3817-3823.

[61] Hsiao T. and Weng M.C. (2013): Robust joint position feedback control of robot manipulators. - J. Dynam. Syst., Measurement, and Control, 135, doi: 10. 1115/1. 4023669.

[62] Davila J., Fridman L. and Levant A. (2005): Second-order sliding mode observer for mechanical systems. - IEEE Trans. Automat. Control, vol.50, No.11, pp.1785-1789.

[63] Atasi A.N. and Khalil H.K. (2000): Separation results for the stabilization of nonlinear systems using different high-gain observer designs. - Systems and Control Letters, vol.39, No.3, pp.183-191.

[64] Levant A. and Livne M. (2012): Exact differentiation of signals with unbounded higher derivatives. - IEEE Transactions on Automatic Control, vol.57, No.4, pp.1076-1080.

[65] Levant A. (2003): Higher-order sliding modes, differentiation and output-feedback control. - International Journal of Control, vol.76, No.9-10, pp.924-941. 